\title{
The design of a vertical curriculum: travelling with mathematics
}

\author{
Antonella Montone ${ }^{* 1}$
}

\begin{abstract}
In this paper the author presents a reflection concerning a possible vertical curriculum in Mathematics. It needs to consider epistemological aspects related to the teaching-learning of math and linked to the suggestions of the Italian National Curriculum. In this case the Vergnaud's Theory of Conceptual Field and the Brousseau's Theory of Situations could prove to be useful for teachers to provide students with fruitful situations so that, facing them, they can construct mathematical knowledge.
\end{abstract}

Keywords: vertical curriculum, Mathematics, teacher.

\section{Introduction}

We live in a constantly evolving society, which is submitted to sudden changes of different kinds and needs more and more appropriate training in employment. The labour market requires rapid changes and specialization becomes a fallacy. Hence the need to create a rational thought, that is also highly flexible and adaptable to the innovations that are constantly taking shape. Therefore, the succession of sudden social and cultural changes have conducted to a careful and deep reflection about the vertical mathematics curriculum.

In particular, the aim is to arrive at what Italian National Curriculum suggest, through the concepts (Indicazioni Nazionali, 2012)2:

* A. Montone, Department of Mathematics, University of Bari, Italy, e-mail: antonella. montone@uniba.it.

1 The part concerning the Theoretical framework of this article has been edited by Antonella Montone and Eleonora Faggiano (Department of Mathematics, University of Bari, Italy).

http://www.indicazioninazionali.it/documenti_Indicazioni_nazionali/indicazioni_ nazionali_infanzia_primo_ciclo.doc. 
His mathematical and scientific-technological knowledge allow him to analyze data and facts based on real life and verify statistics and quantitative analysis proposed by others. His rational thought enables him to face problems and situations based on defined aspects. It gives him the possibility to be aware of the limits of the affirmations that refer to complex issues, which have not unique explanations (...) He has good digital skills and consciously uses communication technologies to seek and analyze data and information, distinguish reliable information from that in need for closer examination, control and verification, and to interact with different people all over the world. He has a fund of knowledge and basic concepts and is able to seek and quickly obtain new information at the same time. He can also learn new things independently (Indicazioni Nazionali, 2012. Authors' translation).

The experience of the first half of 2000's only focused on disciplinary skills missing to highlight the curriculum structure in relation to other elements that Italian National Curriculum had successively pointed out. They had specified attitudes and searches of meaning which are based on historical and cultural evolutions of the more general training of the child and the person.

In recent years, we experienced a smart attempt to connect the Italian National Curriculum in a vertical view especially when referring to the development of skills, from kindergarthen school, to primary and secondary school. This attempt states that:

"The student can independently and responsibly start to face the typical life situations of his age, reflecting and expressing his own personality in all its aspects. This happens at the end of the first school cycle, through school lessons, personal studying, family, and community educational experiences. He is aware of his own potential and limits, uses tools of knowledge to understand himself and the others... he interprets symbolic and cultural systems of society; he chooses consciously, he follows the shared rules, operates with other people for the common good expressing his opinions and sensitivity". This part is complement with we have already said before, in which the importance of mathematics is underlined. It continues: "his mathematical and scientific-technological knowledge allow him to analyze data and facts based on real life and verify statistics and quantitative analysis proposed by others. His rational thought enables him to face problems and situations based on defined aspects. It gives him the possibility to be aware of the limits of the affirmations that refer to complex issues, which have not unique explanations" and it ends with "he shows originality and sense of initiative. He assumes his own responsibilities and asks for help when he is in difficulty and offers help to whom need it. He is willing to analyze himself and face future need and unforeseen events" (Indicazioni Nazionali, 2012, Authors' translation).

This attempt has underlined all the criticality elements referring to some conceptual and rational thought development lacks; which are verified through 
a declarative modality we can call "mathematical behavior". This attempt is verified with a tool called "Invalsi tests"3.

We would like explaining how I said before, through two Invalsi tests examples and their results. In the following example there is a question assigned to last year primary school children.

Figure $1-{ }^{4}$ An example coming from Invalsi test

D23. Quale delle seguenti operazioni dà il risultato più grande?
A. $\square 10 \times 0,5$
B. $\square 10 \times 0,1$
C. $\square 10: 0,5$
D. $\square 10: 0,1$

\section{Risposta corretta: $D$}

RISULTATI DEL CAMPIONE

\begin{tabular}{|c|c|c|c|c|}
\hline A & B & C & D & Non risponde \\
\hline 71,2 & 4,9 & 10,0 & 10,8 & 2,2 \\
\hline
\end{tabular}

This first example shows, not only a lowest percentage of correct answers (only $10.8 \%$ ), but especially a mathematical behavior due to a rigidity of thought, in which the biggest result is obtained through the multiplication rather than a division. And also in this case, 0.5 is bigger than 0.1. This underlines the presence of misconcepts, which are, in this specific case, specific wrong convictions connected to multiplication and division concepts and to rational numbers. In mathematics and in other subjects too, these misconcepts develop in students thought structures. Furthermore, from the analysis of many items referring to thematic field of algebra and arithmetic, there are big similar difficulties and mistakes the same nature.

Also in geometric field, this situation does not change. In fact, it is clear in the following example, during the last year of lower secondary school Invalsi tests.

\footnotetext{
${ }^{3}$ https://invalsi-areaprove.cineca.it/.

${ }^{4}$ Authors' translation: "Which of the following operations gives the biggest result?".
} 
Figure 2 - Geometric example ${ }^{5}$

E6. In figura è rappresentato il rettangolo $A B C D$ con le sue diagonali. Se conosci l'area del rettangolo, puoi calcolare l'area del tríangolo in grigio?

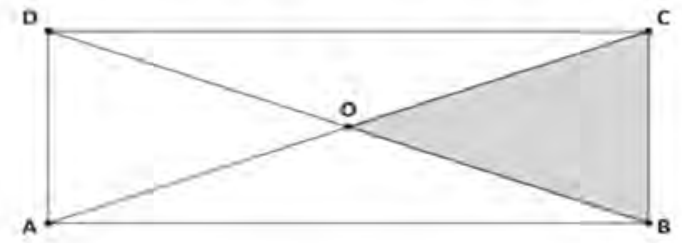
A.
No, perché i quattro triangoli di vertice O non sono tutti uguali fra loro
B.
No, perché non conosco le dimensioni del rettangolo
c.
Si, perchè i quattro triangoli di vertice $O$ sono equivalenti
D. Si, perché i quattro triangoli di vertice $O$ sono isosceli

RISULTATI DEL CAMPIONE

\begin{tabular}{|c|c|c|c|c|}
\hline A & B & C & D & Non risponde \\
\hline 26,9 & 30,8 & 24,1 & 16,4 & 1,6 \\
\hline
\end{tabular}

In this case, students confuse a size with a measurement of quantity. Moreover, they are not able to identify in some geometric properties, for example rectangle diagonals, the useful tool to identify the correct answer. A difficulty connected to a rigorous pattern is again verified; in which some referring to geometric figures problems exclusively concern the calculus of area or perimeter; while the comparison between figures with the same surface only happens through numeric way.

Another proposal has a big diffusion on the national territory, is the Rally Matematico Transalpino (RMT). It concerns many math problems proposed by teachers to a group of students. They are proposed as a mathematical competition (http://smfi.unipr.it/it/orientamento/rally-matematico-transalpinopr). These math problems underline some examples, which show certain similar criticalities and solution modalities.

${ }^{5}$ Authors' translation: E6. The figure shows the rectangle $A B C D$ with its diagonals. If you know the area of the rectangle, can you calculate the area of the gray triangle?

$A$. No, because the four triangles with vertex in $O$ are not all the same

$B$. No, because I do not know the size of the rectangle

C. Yes, because the four triangles are equivalent

D. Yes, because the four triangles are isosceles 
For example, the following problem seems very meaningful to underline a "typical" mathematical behavior connected to wrong convictions about math problems. This is proposed to third, fourth and last primary school students.

Four Rossi children have had a different dessert at the end of lunch. Sonia and her twins did not want the strawberry ice cream. Cecilia has dunked the finger into her sister's caramel pudding. Bernardo, the smallest brother, has found this really funny. One of two males poured a part of his chocolate cream while he was fighting with his brother. What is the dessert has Federico ate? Who ate the apple pie? (RMT).

This problem is part of conceptual logic field. It requires assuming a mathematical behaviour in which it is necessary to:

- Get acquainted of family and desserts;

- Organize information through a table, pattern, and "children-dessert" couples list;

- Find the richest information, and formulate hypotheses and verified them;

- Formulate an explanation and the justification phrases like: "Bernardo is the smallest, and so he is not a twin; he has ate the strawberry ice cream and Federico has ate the chocolate cream..." or through commented table or a drawing.

Below, there is one of the many children examples (in this case it refers to a fourth year primary school children), in which the mathematical behaviour follows a stereotyped pattern, typically scholastic, according to which in a math problem needs localize numeric data, recognize all the math operations to use with different numbers, and resolve it through a block diagram.

From these remarks, the hard questions come from far and they continue to be very current, in spite of all the change and modernity elements already underlined. According to Vergnaud, (1988) we ask:

How does student learn and develop his own ideas?

How can teachers improve their teaching basing on the student learning? (Vergnaud, 1988)

The answers to these questions need of a fundamental introduction to the mathematic planning and didactic experimentation, according to Italian National Curriculum (2012) and also it said before. It is fundamental for every teaching to discipline a cultural theoretical framework to refer to. The a priori analysis of own didactic action, the choice of tools and methodologies to use, the organizing and development activities aim to building math significances during verifying them.

Choosing what to do, assumes also establishing what not to do! 
Figure $3-{ }^{6}$ a solution by a group of 4 student of fourth grade

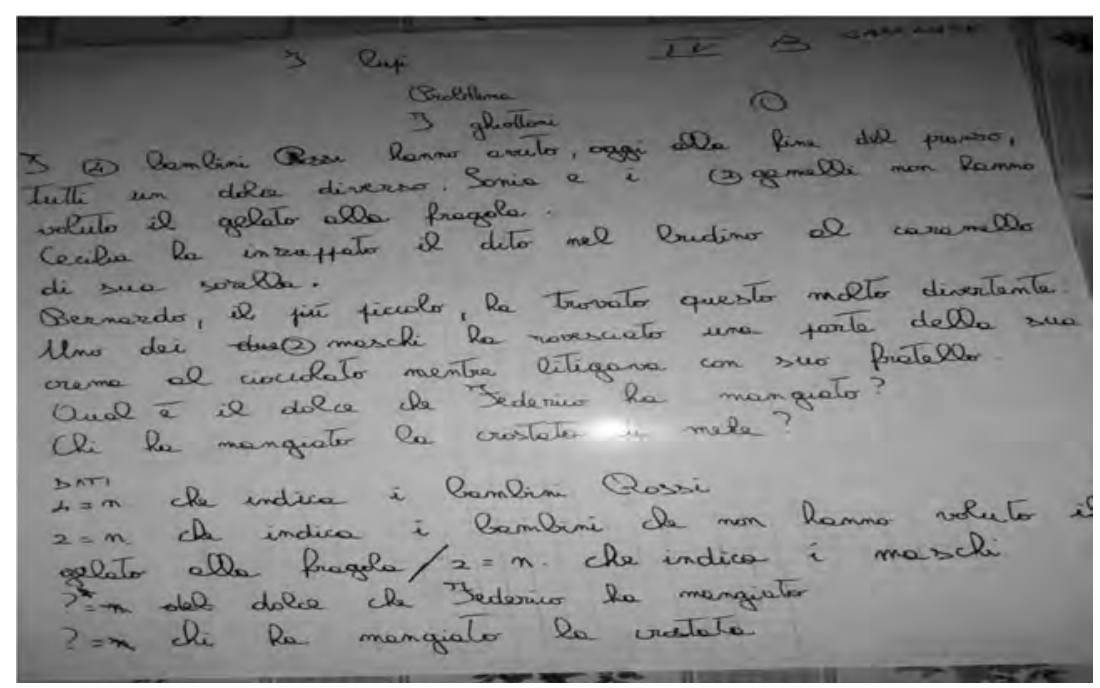

For example, the right using of textbook is a fundamental moment of this choice. Generally, the textbook is a handbook, completed by all mathematic concepts also included into Italian National Curriculum (2012). However, the textbook may not be necessarily prescriptive.

Our main assumption is that, to improve the development and implementation of any curriculum, teachers need to organize didactic situations and interventions, depending on both the epistemology of mathematics and a better understanding of the conceptualizing process of students. Understand how students develop their own mathematical ideas, learn, or fail to learn, mathematics is an essential part of the expertise of teacher and it is the first necessary step of her job. Subsequently, the role of the teacher mainly consists in helping students to develop their repertory of schemes and representations

${ }^{6}$ Authors' translation: "Four Rossi children have had a different dessert at the end of lunch. Sonia and her twins did not want the strawberry ice cream. Cecilia has dunked the finger into her sister's caramel pudding. Bernardo, the smallest brother, has found this really funny. One of two males poured a part of his chocolate cream while he was fighting with his brother. What is the dessert has Federico ate? Who ate the apple pie?"

DATA

$40=\mathrm{n}$ number indicating the Rossi children

$2=\mathrm{n}$ number that indicates the children who did not want strawberry ice cream $/ 2=\mathrm{n}$ number that indicates men

$?=\mathrm{n}$ dessert that has ate Federico

$?=\mathrm{n}$ who ate the apple pie 
by means of which they become able to face more and complex situations (Vergnaud, 2013).

\section{Theoretical framework}

The choice of situations, that cannot be adequate if the teacher does not have a reasonable view and understanding of the involved conceptual field (whose idea will be clarified below), is only the first mediation acts of the teacher. But there are also other mediation acts, such as drawing attention of the students to the relevant properties and relationships, expressing the new knowledge acquired in natural language and other symbolic systems of representation, or helping students to make inferences all along the process.

At this point it is already evident, anyway, that the managing of the longterm process of conceptualization in mathematics implies a large variety of situations, procedures and symbols and for this reason it is particularly difficult.

In all this claims we refer essentially to the Theory of Conceptual Fields developed by Vergnaud $(1988 ; 2009)$ and to the Theory of Didactical Situations developed by Brousseau (1997), on which we are going to deepen ahead. These two theoretical frameworks are useful to help teachers provide students with fruitful situations so that, facing them, they can construct mathematical knowledge.

First of all, it is noteworthy that, according to Vergnaud, (1988) mathematical knowledge is constructed around concept, but a concept is not a mere definition. It is rooted in a set of different situations, involves a set of different operational invariants, and needs to be represented by different linguistic and non-linguistic symbolic elements (Vergnaud, 1988). A conceptual field can be defined as a formal and heterogeneous set of problems, situations, concepts, relationships, structures, contents, and operations interconnected and presumably interwoven during the acquisition process. It is helpful to attribute meanings to acquisition problems and to observations related to conceptualization. Its development involves situations, schemes and symbolic tools of representation.

It is strictly necessary to pay attention to the conceptual aspects of the schemes and to the conceptual analysis of the problem situations for which learners develop their schemes, both in formal teaching and in everyday life. It also important to clarify that in Vergnaud's view a scheme is the invariant organization of behaviour for a certain class of situations, whose essential components are the theorems-in-action - defined as propositions which are held to be true - and the concepts-in-action - defined as objects, predicates or categories which are held to be relevant (Vergnaud, 1998). 
In order to explain these ideas, Vergnaud (1988) starts observing that to give the cardinality of a discrete set of elements most of five or six yearsold children feel the needs to repeat the last word-number (i.e. "one, two, three... six - SIX!"), thus summarizing the information on the set gathered by the counting procedure. The existence in the children of the implicit ideas of one-to-one correspondence and cardinal as invariant property of discrete sets, relying upon an invariant organization - namely scheme - of counting behaviour, can be inferred from this children's behaviour. That is, the scheme of counting a set, which involves the temporal coordination of visual focus, pointing gestures, and recitation of the list of number names, and repetition of the last word-number, is not only made by rules of production but also of operational invariants whose scope of validity can progressively be strengthened and extended.

The implicit knowledge upon which a mathematical behaviour relies and that is contained in schemes is made of concepts and theorems that are used in action, "without being clearly identified and worded as objects, properties and relationships" (Vergnaud, 1988, p. 33). One of the aims of mathematical education is to build up explicit and general concepts and theorems from the usually implicit concepts-in-action and theorems-in-action involved in the understanding of new situations. Nevertheless, if the role of the schemes in the development of knowledge is essential, it is also relevant that linguistic and symbolic expressions play an important part in mathematics and in mathematics education because they are necessary to communicate to others and to discuss. Operational invariants can be transformed into categories and sentences and the conceptualization can move towards higher level objects and predicates, thus changing the status of knowledge to explicit.

In agreement with Vergnaud (2009), we can say that to gain sense and operability, a concept's meaning needs to be contextualized and exemplified in situation. However, it does not come from one situation only but from a variety of situations and, reciprocally, it is not possible to analyse a given situation with the use of only one concept but rather with several concepts, forming systems. As a consequence, the construction and appropriation of all the properties of a concept, or of all the aspects involved in a situation, are comprehensive and lengthy processes which require students' meeting and being faced with contrasting situations. For this reason, as it is fruitful for the teacher to imagine/chose situations likely to push and help students to move along the multifaceted complexity of the field, it is clear that teachers need to be aware of the characteristic of at least the main mathematical conceptual fields.

If the long-term development of mathematical competences and conceptions requires the teacher to understand connections and jumps involved, Brousseau's 
Theory of Didactical Situations (TDS) (Brousseau, 1997 can be considered as a useful framework to provide adequate situations.

According to Brousseau (1997), learning is considered as resulting from an adaptation to a new situation in which the student's prior knowledge does not allow him/her to develop an efficient solving strategy and so he/she must develop new means of solution that are the source of new mathematical knowledge. Therefore, the focus is on the situations as leading to learning a particular piece of knowledge. The goal for the teacher should be to identify appropriate situations in order to support a process of guided reinvention in which the students develop a scientific activity that can be the source of the construction of knowledge. Brousseau explains what such a process involves and requires when he writes that: "[a] faithful reproduction of a scientific activity by the student would require that she produce, formulate, prove, and construct models, languages, concepts, and theories; that she exchange them with other people" (Brousseau, 1997, p. 22).

More precisely, he defines an a-didactical situation to be a situation in which the student is enabled to use some knowledge to solve a problem "without appealing to didactical reasoning [and] in the absence of any intentional direction [from the teacher]" (ibid., p. 30). Indeed, what characterizes the a-didactical situation - the only one through which true knowledge acquisition can be said to happen - is the fact that it is "partially freed from the teacher's direct interventions" (Brousseau, 2003, p. 2). When referring to it, Brousseau underlines that "between the moment the student accepts the problem as if it were her own and the moment when she produces her answer, the teacher refrains from interfering and suggesting the knowledge that she wants to see appear" (Brousseau, 1997, p. 30). The teacher's enterprise, therefore, is to organize the devolution of an a-didactical situation to the learner, where for devolution Brousseau means the act by which the teacher makes the student accept the responsibility for an a-didactical learning situation or for a problem, and accepts the transfer of this responsibility.

A didactic situation is a set of explicit and implicit relationships established between a student or a group of students, some padding (tools and materials too), and the teacher in order to allow students to learn. In other words, they learn, or, rebuild some of their knowledge. Situations are specific of this knowledge.

\section{Operative proposal: from conceptual fields to the thematic core}

An example made by Vergnaud, (1988) in the literature, efficiently shows how the same scheme, which is an invariant organization of mathematic 
behaviour, can be associated to a different class of situations, expressed through the three following problems:

1. Pietro had 5 marbles. He plays a game of marbles with John and wins 7 marbles. How many marbles does he have now?

2. Robert has just played a game of marbles with Celia. He had lost 7 marbles. He has now 5 marbles. How many marbles did he have before playing?

3. John has played two games of marbles. He has lost 7 marbles in the second game, but he does not remember what happened in the first game. When he counts his marbles in the end, he finds that he has altogether won 5 marbles. What happened in the first game? (Vergnaud, 1988, p. 33)

The solving scheme provides for the addition " $5+7$ " in these problems. However, they shall implement different mathematical behaviors that are connected to reasoning modalities and are developed during a long timeframe. This is connected to the development of the conceptual field of additivity. As highlighted by Vergnaud, the first problem refers to primitive conception of addition understood as an increment. Thus, it is defined on all the Natural Numbers. Instead, the second problem requires an inversion of transformation that leads -7 to become +7 . In other words, a transformation from the final status to the initial status is used, reversing the natural and intuitive transformation from an initial status to a final status. Since the text shows the expression "lost", the addition that resolves the problem is against the primitive conception (nothing is added when marbles are lost). Generally, mathematicians and teachers understand the addition as an internal binary composition commutative law. This creates one part that represents the total starting from two parts. Instead, subtraction is conceived as the research of a part knowing the total and the other part.

However, the psychological classification of cognitive problems admits the existence of a unary operation, which corresponds to the transformation of the initial status. The relation below the first problem is "initial statustransformation- final status", while this model cannot count for the second problem. A not commutative unary operation is necessary, which is able to invert the previous transformation. Moreover, the "numbers with a sign" are necessary; they have value in all transformations called "negative" (i.e.: he lost seven marbles (-7), I ate three candies (-3), temperature dropped ten degrees $(-10)$, and so on).

In the third problem, the addition $5+7$ is completely out of the intuition. In this situation, it is necessary to add all the parts, the unknown result of round one (x), the marbles lost during round two $(-7)$, to obtain the other part. The two parts are considered as partis of a composed transformation that connects the initial status to the final status. 
If Giovanni had lost 15 marbles, the answer would had been easy and natural. However, the third problem requires an addition, $5+7$, which is actually a subtraction between two relative numbers

$\mathrm{x}+(-7)=(+5)$

$\mathrm{x}=(+5)-(-7)=5+7=+12$

The conceptual fields, is that of additive structures within elementary calculus (Vergnaud, 1988). It comprises the contexts and situations for which additive structures (addition and subtraction) are required. This field includes counts, measurement and situations of comparison; numbers with the related concept of order and equality; and addition and subtraction operations, including their properties, as well as their relationships with order relations and with classes of problems that use these operations for their solution.

\section{The role of the teacher}

In common belief, one often thinks that addition is an operation that starts and ends during primary school, and that what has been learnt (operation idea and calculus algorithm between natural numbers) must be only transferred to other numeric systems.

Instead, it is very important to keep in mind that addition is an operation included in the conceptual field of additive structure. Its sense ends during secondary school when other concepts and other fields strictly connected to it, such as algebra and real numbers, are introduced. The school period from 4 to 15 years old provides a cognitive development during which students discover and learn to solve a great variety of problematic situations. Conceptual field of additive structures involves many important concepts, which often are implicit for students, as stated above.

Therefore, during the long school period, the teacher also assumes the fundamental role of the one who mediates the construction of meanings and makes concepts explicit, through "collective debates" (Bartolini, 1998). Moreover, during this long period, the symbols and language roles in the formation of concepts and solving of problems that are connected with the tough thought operations required, should not be underestimated.

In this context, it is relevant introducing the first elements that are related to algebra language intended as translation and generalization of situations in Primary School. Algebra represents one of thematic cores that more than others creates difficulties and often a definitive break-up between students and maths. One of the reasons is the lack of comprehension due to the generality of algebra expressions. 
Beyond that of algebra structures, other examples of conceptual fields are those of multiplicative and geometric structures.

We think that the idea of conceptual field lend itself very well to the formulation of a vertical curriculum, because it widens and enriches in a very long timeframe that is strictly connected to the period of students intellectual growth. What is more, the first mathematical learning of primary school students is of greatest importance and relevance in this field. To this end, another relevant element is the a priori analysis of problems that teachers could use in the elaboration of didactic situations. This analysis individualizes the conceptual circle in which the problem is inserted and the mathematical aspects that can be found in the Italian National Curriculum (2012), to which the problem tightly connects. Moreover, it allows to deeply understand the meaning of conceptual field and how the problem ties in a particular field, in order to individualize mathematical concepts that are needed first and those that could develop after.

The teacher ability to analyse and predict about students' mathematical behaviours also departing by his own, becomes a significant element to assume the development of a conceptual field in the didactic practice.

Furthermore, these analyses need an interconnection between teachers of different grades. Only the cooperation and confrontation between teachers with different skills and experiences can guarantee an organization and realization of a vertical mathematical curriculum that is exhaustive and coherent with the didactic theories presented.

\section{Conclusions}

Italian National Curriculum (2012) represents an inexhaustible source of didactic suggestions and a precious resource for teachers to face the big challenge of creating a vertical mathematical curriculum too. Furthermore, every didactic design must refer to a theoretical framework and epistemological aspects related to the teaching-learning of math.

Only a proposal that can destabilise the student through new and motivating problematic situations to push him to discover and learn new concepts and take account of the implications suggested by theoretical frameworks (Conceptual Fields and Situations theory) can be a part of a broad vision in a very long period characterising a vertical curriculum. 


\section{References}

Bartolini Bussi, M.G. (1998). Verbal interaction in mathematics classroom: A Vygotskian analysis. In H. Steinbring, M.G. Bartolini Bussi, \& A. Sierpinska (eds.), Language and communication in mathematics classroom (pp. 65-84). Reston, VA: NCTM.

Brousseau, G. (1997). Theory of Didactical Situations in Mathematics. Dordrecht: Kluwer.

Brousseau, G. (2003). Glossaire de quelques concepts de la théorie des situations didactiques en mathématiques. Retrieved from http://dipmat.math.unipa.it/ grim/ Gloss_fr_Brousseau.pdf.

Indicazioni Nazionali per il Curricolo, 2012 http://www.indicazioninazionali.it/ documenti_Indicazioni_nazionali/indicazioni_nazionali_infanzia_primo_ciclo.doc. https://invalsi-areaprove.cineca.it/.

Rally Matematico Transalpino (RMT), http://smfi.unipr.it/it/orientamento/rallymatematico-transalpino-pr.

Vergnaud, G. (1988). Theoretical frameworks and empirical facts in the psycology of mathematics education. Plenary address in Ann \& Keith Hirst (eds.), Proceedings of ICME 6, pp. 29-47.

Vergnaud, G. (1998). A Comprehensive Theory of Representation for Mathematics Education. Journal of Mathematical Behaviour, 17 (2), pp. 167-181.

Vergnaud, G. (2009). The Theory of Conceptual Fields. Human Development, 52, pp. 83-94.

Vergnaud, G. (2013). Conceptual development and learning. Revista Qurriculum, 26, pp. 39-59. 